\title{
Aplicación de un Algoritmo Evolutivo en la Solución de Problemas Job Shop-Open Shop
}

\author{
Omar D Castrillón, William A Sarache y Jaime A Giraldo \\ Universidad Nacional de Colombia, Campus la Nubia. Manizales-Colombia \\ (e-mail: odcastrillong@unal.edu.co,wasarachec@unal.edu.co, jaiagiraldog@unal.du.co)
}

Recibido Abr. 21, 2010; Aceptado Jun. 17, 2010; Versión Final recibida Ago. 01, 2010

\begin{abstract}
Resumen
El objetivo del trabajo que se presenta fue disminuir el tiempo de proceso y el tiempo muerto, y aumentar la utilización de las máquinas, en un ambiente Job Shop-Open Shop, usando una nueva metodología basada en un algoritmo evolutivo. El estudio se realizó en una empresa del sector metalmecánico. La metodología propuesta es fácil de replicar y los resultados obtenidos son altamente consistentes, como se demuestra con un análisis de varianza realizado. Con la metodología propuesta se logró reducir el tiempo total de proceso en un 33\% y el tiempo muerto en un 51\% con una aproximación del 99\%, respecto a la solución óptima estimada.
\end{abstract}

Palabras clave: job shop, open shop, algoritmo evolutivo, tiempo de proceso, tiempo muerto

\section{Application of an Evolutionary Algorithm to solve Job Shop-Open Shop Problems}

\begin{abstract}
The aim of the work presented in this paper was to reduce makespan time and idle time, and to increase machine utilization, in Job Shop-Open Shop environment, using a new methodology based on evolutionary algorithms. The study was done in an enterprise of the metal-mechanics sector. The proposed methodology is easy to implement and apply and the results are highly consistent, as shown by a variance analysis. The methodology allows reducing the total processing time by $33 \%$ and idle time by $51 \%$ with $99 \%$ approximation with respect to the optimum solution.
\end{abstract}

Keywords: job shop, open shop, evolutionary algorithm, makespan time, idle time 


\section{INTRODUCCIÓN}

Actualmente las técnicas de Inteligencia Artificial, han sido ampliamente empleadas en la solución de una gran variedad de problemas en la industria y el comercio (Weiming, et ál. 2006), la agricultura (Nebendahi. 2000), la programación de la producción, construcción de ordenes de producción (López, et ál. 2009), el diseño de productos, la planificación de inversiones, la navegación marítima (Rancán. 2004), la disminución del riesgo y tiempo experimental, la incorporación y fusión de resultados de modelos de simulación de diferentes fuentes y disciplinas, la automatización de edificios (Sierra, et ál. 2005) la planificación de sistemas, la secuenciación de la producción en ambientes Job Shop (Nilgun, et al. 2009; Adil, et ál. 2009 ), la optimización de procesos (Zhang, et ál. 2009), la minimización de tiempos de proceso (Pei-Chann, et ál. 2009; Roshanaei, et ál. 2009; Louis, et ál. 1996), simulación (Perez. 2004; Rosa. 2004) y en general toda clase de aplicaciones (Savkin, et ál. 2009; De Giovanni, et ál. 2009; Chao-Hsien, et ál. 2009; Castrillon, et ál. 2008), (Leung. 2004; Latta, et ál. 2000, Anaut. 2009).

Específicamente, en la solución de problemas Job Shop - Open Shop se han empleado diferentes técnicas de inteligencia artificial, como se ilustra en la Tabla 1.

Aunque, en la Tabla 1, se ilustra una gran la variedad de metodologías basadas en inteligencia artificial para la solución de problemas Job Shop - Open Shop, este artículo tiene como objetivo fundamental combinar varias de las principales características ilustradas en las metodologías existentes y diseñar por medio de un algoritmo evolutivo, una nueva metodología (para la solución de estos problemas), con una efectividad y robustez superior al $99 \%$ y un tiempo de computo prácticamente despreciable. Esta metodología deberá optimizar u sub optimizar las variables tiempo total de proceso (Makespan) y tiempo total muerto (time idle) en la solución de un problema de secuenciación de la producción en un ambiente Job Shop - Open shop; un problema donde el tiempo total muerto es una consecuencia directa del tiempo total de proceso.

El anterior aspecto hace que esta metodología tenga un espacial interés no solo para la industria, sino también para todos los profesionales en el área de la ingeniería, gremios, directivos empresariales y en general para toda la comunidad.

La metodología fundamentalmente se estructura en los siguientes pasos: a) definición matricial de un problema Job Shop. (Koonce. 2000) b) construcción de las diferentes soluciones iníciales. c) evolución de las soluciones iníciales hasta alcanzar una condición de parada, según los valores estipulados por el tiempo total de proceso y el tiempo total muerto. d) Estimación del tiempo de proceso óptimo y determinación del porcentaje de aproximación de las soluciones encontradas respecto a la solución óptima estimada e) cálculo de consistencia de las mejores soluciones generadas, por medio de un análisis de varianza. f) comparación con las metodologías tradicionales de programación de la producción y con otras metodologías evolutivas.

Igualmente, es importante resaltar que en estos problemas es muy difícil encontrar una solución óptima, especialmente cuando el número de pedidos $\mathbf{N}$ y el número de centros de trabajo $\mathbf{M}$, es demasiado grande, dado que las soluciones posibles están determinadas por la expresión $\mathbf{M} !^{\mathrm{N}}$; (Leung. 2004). En consecuencia se hace necesario estimar una solución óptima, sobre la cual se aproximaran los resultados de la nueva metodología diseñada en este artículo.

Es importante expresar que las soluciones encontradas, por medio de esta nueva metodología, se aproximan en un porcentaje superior al 99\%, respecto a la solución óptima estimada. Un resultado superior, en un $66 \%$, a los resultados obtenidos con las metodologías tradicionalmente empleadas como operación más corta $(O M C)$, operación más larga $(O M L)$, pilas $(F I F O)$, colas (LIFO), Campbell. (Domínguez, et ál. 1995). 
Tabla 1. Técnicas de Inteligencia Artificial en la solución de problemas Job Shop - Open Shop.

\begin{tabular}{|c|c|c|c|}
\hline Método /Estudio & Tipo de Estudio & Comentario & Referencia \\
\hline $\begin{array}{l}\text { Open shop scheduling } \\
\text { and re-scheduling. }\end{array}$ & $\begin{array}{l}\text { Algoritmos genéticos y } \\
\text { un sistema de } \\
\text { razonamiento basado en } \\
\text { casos. }\end{array}$ & $\begin{array}{l}\text { Muestra una evolución } \\
\text { de la función makespan, } \\
\text { sin establecer } \\
\text { comparaciones con un } \\
\text { óptimo general. }\end{array}$ & (Louis, et ál 1996) \\
\hline $\begin{array}{l}\text { Open - Shop con dos } \\
\text { máquinas. }\end{array}$ & $\begin{array}{l}\text { Se emplea programación } \\
\text { dinámica en la solución } \\
\text { de este problema. }\end{array}$ & $\begin{array}{l}\text { Se llega a una solución } \\
\text { en un tiempo } \\
\text { pseudopolinomial. }\end{array}$ & (Strusevich, 1997) \\
\hline $\begin{array}{lr}\text { Open - Shop con dos } \\
\text { máquinas } & \text { y } \\
\text { restricciones. } & \end{array}$ & $\begin{array}{l}\text { Diseño de un algoritmo } \\
\text { de aproximación. }\end{array}$ & $\begin{array}{l}\text { Se soluciona este } \\
\text { problema considerando } \\
\text { que una maquina no está } \\
\text { disponible durante un } \\
\text { tiempo de proceso } \\
\text { determinado. }\end{array}$ & (Joachim, 2001) \\
\hline Open Shop. & Colonia de hormigas. & $\begin{array}{l}\text { Se diseña un mecanismo } \\
\text { de construcción } \\
\text { probabilística como } \\
\text { método de búsqueda de } \\
\text { arboles. }\end{array}$ & (Christian, 2005) \\
\hline $\begin{array}{l}\text { Tiempo medio de flujo } \\
\text { en un problema open } \\
\text { shop. }\end{array}$ & $\begin{array}{l}\text { Recocido simulado y } \\
\text { algoritmos genéticos. }\end{array}$ & $\begin{array}{l}\text { Para solucionar cada } \\
\text { problema JSSP, con } \\
\text { cada una de las técnicas, } \\
\text { se emplean } 30.000 \\
\text { iteraciones. }\end{array}$ & (Michel, et ál. 2008) \\
\hline $\begin{array}{l}\text { Programación de tienda } \\
\text { abierta. }\end{array}$ & $\begin{array}{l}\text { Enjambre de partículas. } \\
\text { (PSO). }\end{array}$ & $\begin{array}{l}\text { Modifica el algoritmo } \\
\text { PSO tradicional, con el } \\
\text { uso de prioridades. No } \\
\text { se establecen } \\
\text { comparaciones con una } \\
\text { solución óptima. }\end{array}$ & (Dy, et ál. 2008) \\
\hline $\begin{array}{l}\text { Problemas Open - } \\
\text { Shop. }\end{array}$ & $\begin{array}{l}\text { Programación entera y } \\
\text { algunas técnicas } \\
\text { heurísticas. }\end{array}$ & $\begin{array}{l}\text { Esta técnica permite } \\
\text { encontrar soluciones con } \\
\text { una efectividad superior } \\
\text { al } 99 \% \text {. En un tiempo de } \\
\text { cómputo razonable. }\end{array}$ & (Chianyao, et ál. 2009) \\
\hline $\begin{array}{l}\text { Solución de un problema } \\
\text { Job Shop - Open Shop. }\end{array}$ & $\begin{array}{l}\text { Aplicación de un } \\
\text { algoritmo evolutivo en la } \\
\text { solución de un problema } \\
\text { JSSP } 15 \times 20 \text {. }\end{array}$ & $\begin{array}{l}\text { Bajo una nueva } \\
\text { metodología se } \\
\text { establecen soluciones } \\
\text { con una efectividad y } \\
\text { robustez superior al } \\
99 \% \text {. Este algoritmo } \\
\text { emplea un máximo de } \\
500 \text { iteraciones. }\end{array}$ & Este artículo (2010) \\
\hline
\end{tabular}

\section{MATERIALES Y METODOS}

\section{Metodología}

Inicialmente, la metodología propuesta, empieza por considerar los supuestos, propuestos en Rohrer (2000), posteriormente la misma es definida como se ilustra a continuación:

Paso 1. Definición: El problema JSSP es representado por medio de una matriz, donde el número de la fila representa el centro de trabajo y el número de la columna, representa el pedido. Cada uno de los valores (Fil, Col) representa el tiempo de proceso del pedido i en la maquina $\mathrm{j}$. (Koonce. 2000). 
Tabla 2. Representación del problema JSSP NXM.

\begin{tabular}{|c|c|c|c|c|}
\hline & Ped $_{1}$ & Ped $_{2}$ & $\ldots$ & Ped $_{n}$ \\
\hline$C_{1}$ & & $T p$ & & \\
\hline.. & & & & \\
\hline$C_{m}$ & & & & \\
\hline
\end{tabular}

Paso 2. Soluciones iníciales: En es este paso, se deben codificar cada una de las soluciones iníciales en forma de cromosomas, (se generan 10 soluciones iníciales de forma aleatoria y se seleccionan las dos mejores de ellas). Cada uno de los genes que conforman cada cromosoma representa el orden en que un pedido será atendido por cada una de las diferentes máquinas. En cada gen, sus alelos representan el número del pedido (Fig. 1). (Castrillón, et ál, 2010)

\section{Cromosoma}

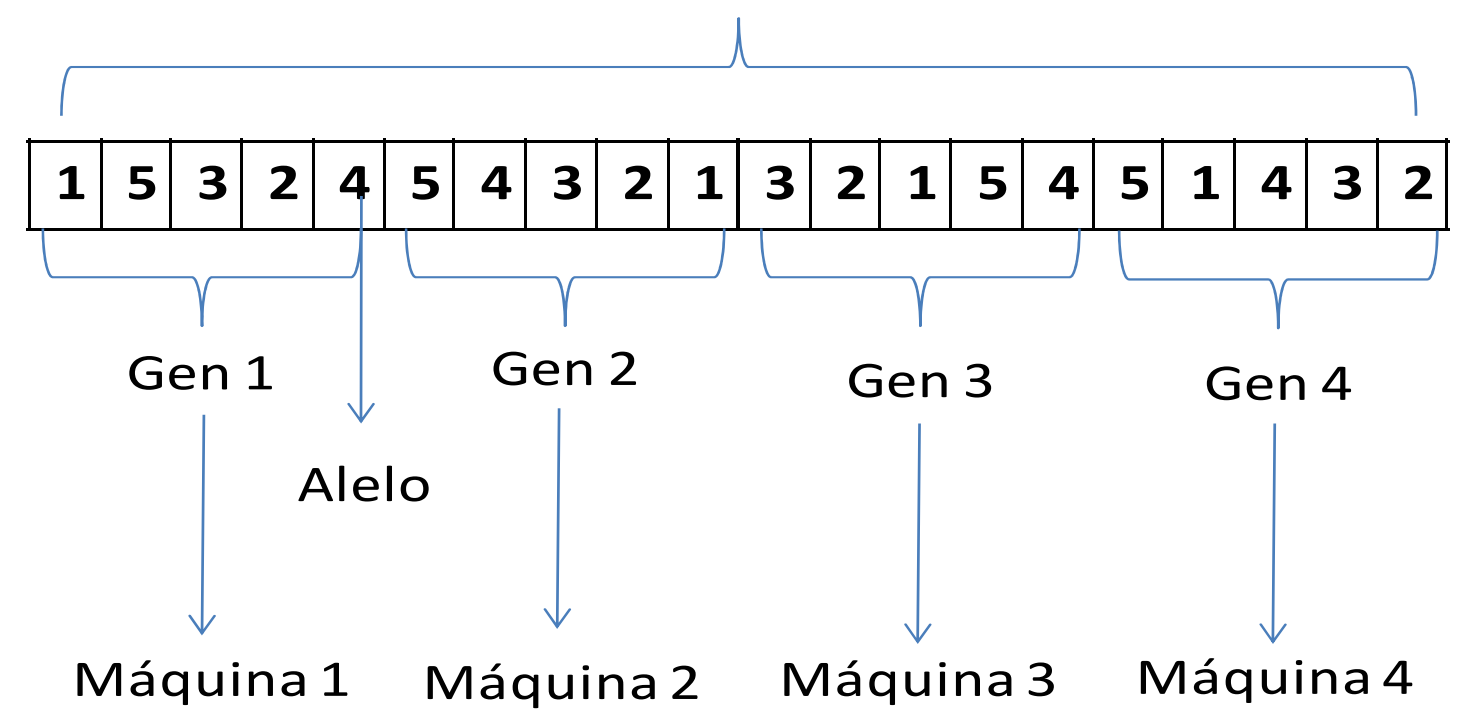

Fig 1: Codificación de las soluciones. Fuente: (Castrillón et ál, 2010)

Paso 3. Evaluación: Para cada una de las soluciones, establecidas en el paso anterior, se debe definir un diagrama de Gantt (para cada solución), el cual establezca el orden de los procesos en el tiempo, en cada uno de los diferentes centros de trabajo. Una vez establecido el anterior diagrama se procede a evaluar cada una de las diferentes soluciones, con el fin de calcular los tiempos totales de proceso (Makespan) y los tiempos totales de ocio (Idle), bajo las siguientes funciones de cálculo (Fitness):

Fitness $_{i d l e}=\min \sum_{j=1}^{M} f_{j}$

Fitness $_{\text {Makespan }}=\min \sum_{i=1}^{N} \sum_{j=1}^{M} P_{i j}$

El objetivo fundamental, es minimizar las dos funciones Fiteness. Donde $\boldsymbol{N}$ representa el número de trabajos. $\boldsymbol{M}$, representa el número de máquinas, $\mathrm{P}_{\mathrm{ij}}$ es el tiempo de procesamiento del trabajo $\boldsymbol{i}$, en la máquina $\boldsymbol{j}$ y $\boldsymbol{f}_{\boldsymbol{j}}$, es el tiempo total ocio de la maquina $\mathbf{j}$. 
Dado que el tiempo muerto (Idle Time) es una consecuencia directa del tiempo de proceso (makespan), la búsqueda solo es guiada por la función makespan Ecuación (2). Con base en el menor tiempo makespan, es calculado el tiempo total muerto, según la ecuación (1).

Posteriormente, el proceso continúa evolucionando con una probabilidad de combinación del 97\% y una probabilidad de mutación del $3 \%$, hasta que se encuentre una solución que se aproxime en un $99 \%$ a la solución óptima estimada (véase paso 4) ó se generen 100 soluciones sin que se mejore el tiempo total de proceso (makespan) o se complete 500 iteraciones. En cada paso de la evolución, solamente se genera un hijo (esquema estacionario).

La nueva población es seleccionada por medio de un operador de ranking, en el cual la probabilidad de selección es directamente proporcional a la aproximación de la solución que representa cada individuo, respecto a la solución óptima estimada, reemplazándose el peor individuo de la población. Para que las soluciones presentadas continúen siendo validas, en cada gen el operador de mutación debe intercambiar de forma aleatoria dos alelos diferentes. (Figura 1). Igualmente el operador de combinación, selecciona las dos mejores soluciones y combina la primera mitad de los genes de una solución con la segunda mitad de los genes de la otra solución (Solo se combinan genes completos).

Paso 4. Estimación del óptimo: Con el fin de calcular la aproximación de las soluciones encontradas, respecto a la mejor solución, es necesario estimar la solución óptima. Para estimar la solución óptima, se considera que el tiempo de proceso óptimo nunca es inferior al máximo tiempo de proceso entre: El máximo tiempo empleado por los centros de trabajo, con tiempo muerto igual a cero y el tiempo de proceso total que emplea un pedido $\mathbf{P}_{\mathbf{i}}$ en pasar por todos los centros de trabajo, con un tiempo muerto igual a cero.

El tiempo óptimo permitirá determinar la efectividad de la metodología propuesta y establecer el porcentaje de aproximación, de cada una de las posibles soluciones encontradas, respecto al óptimo general.

Paso 5. Análisis de varianza: Con el propósito de garantizar la consistencia de la metodología es necesario repetir la misma, durante un determinado número de veces (tratamientos) respecto a la función Fitnes (ecuación 2). Con el fin de determinar si los resultados corresponden estadísticamente a tratamientos iguales o diferentes, un análisis de varianza bajo el siguiente modelo es realizado: $y_{i}=\mu+\mathrm{T}_{i}+\varepsilon_{i}$, donde $y_{i}$ representa las variables de respuesta, $\mathrm{T}_{i}$, los efectos causados por el tratamiento $\mathrm{i}^{\text {th }}, \mathrm{y} \varepsilon_{i}$, es el $\mathrm{i}^{\text {th }}$ error experimental.

En este punto, es importante verificar que la información recolectada cumpla con las condiciones necesarias de independencia y normalidad, que permitan aplicar los test requeridos. (Castrillón, et ál. 2009).

Paso 6. Metodologías tradicionales: Para determinar la efectividad de esta metodología frente a los métodos comúnmente empleados en la programación de la producción, el problema es solucionado mediante las técnicas tradicionalmente empleadas por las empresas: OMC, OML, FIFO, LIFO, Campbell. Los resultados son comparados con el óptimo estimado.

\section{Experimentación:}

En la experimentación de esta metodología, se tomó como referencia una empresa del sector metalmecánico, en su producto fundamental denominado "Barras". Aunque en el problema original, el producto debe pasar por cinco centros de trabajo sin importar el orden; la experimentación se realizó con base en un problema de carácter general, con 15 centros de trabajos y 20 pedidos; restricción realizada, solo por razones computacionales.

Los tiempos de procesos de los diferentes pedidos en cada uno de los diferentes centros de trabajo son ilustrados en la tabla 3 . En esta tabla 3 , la última columna (sum) representa la 
sumatoria de todos los tiempos de proceso de un pedido en un centro de trabajo $\left(C_{i}\right)$. Igualmente, la última fila representa el tiempo de proceso total que emplea un pedido $\mathbf{P}_{\mathbf{i}}$ en pasar por todos los centros de trabajo, con un tiempo muerto igual a cero.

Entonces, como se ilustró en el paso 4 de la sección Materiales y Métodos, el mayor valor entre estos dos valores, puede ser considerado como un óptimo estimado. En este caso es 218.

Tabla 3. Problema objeto de estudio. $15 !^{20}=2.1379 * 10^{242}$ Posibles soluciones.

\begin{tabular}{|c|c|c|c|c|c|c|c|c|c|c|c|c|c|c|c|c|c|c|c|c|c|}
\hline & $\mathbf{P}_{1}$ & $\mathbf{P}_{2}$ & $\mathbf{P}_{3}$ & $\mathbf{P}_{4}$ & $\mathbf{P}_{5}$ & $\mathbf{P}_{6}$ & $\mathbf{P}_{7}$ & $\mathbf{P}_{8}$ & $\mathbf{P}_{9}$ & $\mathbf{P}_{10}$ & $\mathbf{P}_{11}$ & $\mathbf{P}_{12}$ & $\mathbf{P}_{13}$ & $\mathbf{P}_{14}$ & $\mathbf{P}_{15}$ & $\mathbf{P}_{16}$ & $\mathbf{P}_{17}$ & $\mathbf{P}_{18}$ & $\mathbf{P}_{19}$ & $\mathbf{P}_{\mathbf{2 0}}$ & Sum \\
\hline $\mathrm{C}_{1}$ & 1 & 4 & 3 & 2 & 4 & 6 & 8 & 9 & 7 & 5 & 4 & 7 & 7 & 5 & 6 & 8 & 7 & 4 & 1 & 3 & 101 \\
\hline$C_{2}$ & 4 & 1 & 2 & 10 & 8 & 11 & 5 & 3 & 1 & 10 & 2 & 3 & 10 & 6 & 1 & 9 & 3 & 7 & 7 & 8 & 111 \\
\hline $\mathrm{C}_{3}$ & 6 & 1 & 5 & 4 & 7 & 2 & 8 & 6 & 8 & 6 & 3 & 12 & 4 & 3 & 4 & 10 & 12 & 3 & 8 & 1 & 113 \\
\hline $\mathrm{C}_{4}$ & 9 & 3 & 1 & 5 & 1 & 7 & 9 & 1 & 5 & 4 & 8 & 1 & 5 & 9 & 1 & 8 & 5 & 6 & 3 & 2 & 93 \\
\hline$C_{5}$ & 3 & 4 & 5 & 9 & 9 & 5 & 4 & 2 & 4 & 9 & 7 & 6 & 6 & 8 & 10 & 4 & 4 & 3 & 4 & 3 & 109 \\
\hline $\mathrm{C}_{6}$ & 9 & 3 & 1 & 5 & 1 & 7 & 9 & 1 & 5 & 4 & 8 & 1 & 5 & 9 & 1 & 8 & 5 & 6 & 3 & 2 & 93 \\
\hline$C_{7}$ & 15 & 4 & 12 & 2 & 8 & 3 & 8 & 12 & 7 & 5 & 9 & 3 & 9 & 17 & 13 & 32 & 12 & 12 & 9 & 8 & 200 \\
\hline $\mathrm{C}_{8}$ & 9 & 3 & 1 & 34 & 1 & 7 & 9 & 1 & 5 & 4 & 8 & 1 & 5 & 9 & 1 & 8 & 5 & 6 & 3 & 2 & 122 \\
\hline $\mathrm{C}_{9}$ & 1 & 4 & 3 & 2 & 4 & 6 & 8 & 9 & 7 & 5 & 4 & 7 & 7 & 5 & 6 & 8 & 7 & 4 & 1 & 3 & 101 \\
\hline $\mathbf{C}_{10}$ & 17 & 6 & 14 & 4 & 10 & 5 & 10 & 14 & 9 & 5 & 9 & 3 & 9 & 17 & 13 & 32 & 12 & 12 & 9 & 8 & 218 \\
\hline $\mathrm{C}_{11}$ & 6 & 1 & 5 & 4 & 7 & 2 & 8 & 6 & 8 & 6 & 3 & 12 & 4 & 3 & 4 & 10 & 12 & 3 & 8 & 1 & 113 \\
\hline $\mathrm{C}_{12}$ & 9 & 3 & 1 & 5 & 1 & 7 & 9 & 1 & 5 & 4 & 8 & 1 & 5 & 9 & 1 & 8 & 5 & 6 & 3 & 2 & 93 \\
\hline $\mathrm{C}_{13}$ & 4 & 1 & 2 & 10 & 8 & 11 & 5 & 3 & 1 & 10 & 2 & 3 & 10 & 6 & 1 & 9 & 3 & 7 & 7 & 8 & 111 \\
\hline $\mathrm{C}_{14}$ & 9 & 3 & 1 & 5 & 1 & 7 & 9 & 1 & 5 & 4 & 8 & 1 & 5 & 9 & 1 & 8 & 5 & 6 & 3 & 2 & 93 \\
\hline $\mathrm{C}_{15}$ & 1 & 4 & 3 & 2 & 4 & 6 & 8 & 9 & 7 & 5 & 4 & 7 & 7 & 5 & 6 & 8 & 7 & 4 & 1 & 3 & 101 \\
\hline Sum & 103 & 45 & 59 & 103 & 74 & 92 & 117 & 78 & 84 & 86 & 87 & 68 & 98 & 120 & 69 & 170 & 104 & 89 & 70 & 56 & 1772 \\
\hline
\end{tabular}

\section{RESULTADOS}

Paso 1-4: El mejor tiempo de proceso encontrado, mediante la técnica descrita en los pasos 1, 2, 3 y 4 de la metodología propuesta, fue 220 el cual corresponde a un tiempo muerto de 1528 (Figura. 2). Igualmente, la Tabla 2, muestra que el tiempo total de proceso de todos los pedidos en el centro de trabajo 10 es de 218, en consecuencia cualquier solución óptima nunca podrá ser inferior a 218, siendo factible tomar este último tiempo de proceso (218) como el óptimo estimado. Por ende, la mejor solución encontrada, por lo menos se aproxima en $99.1 \%$ respecto a la solución óptima estimada, con un tiempo de computo estimado de 4.2 segundos. 


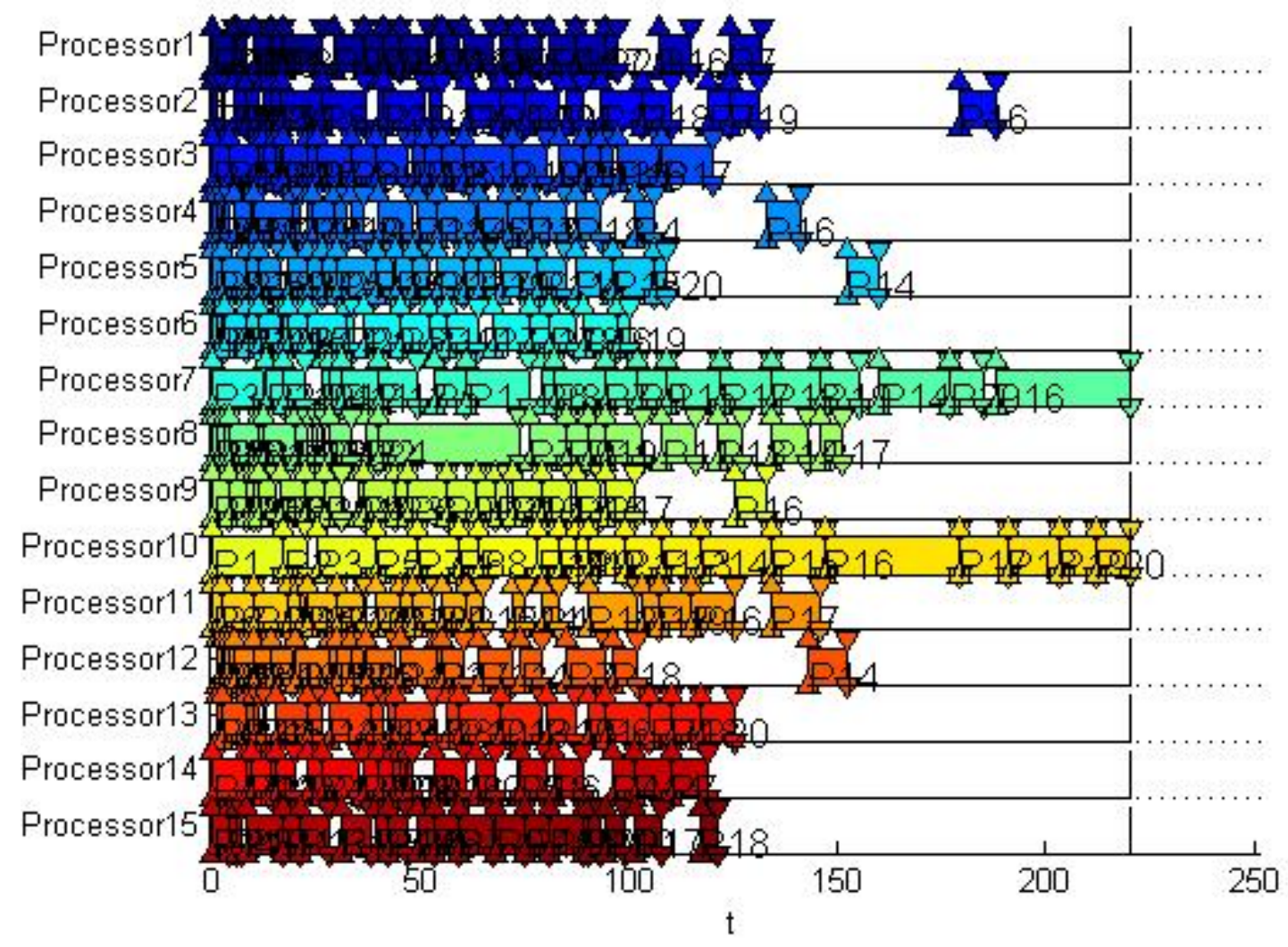

Fig 2. Mejor Solución encontrada. Tiempo de proceso $=220$, Tiempo Muerto $=1528$

Paso 5: Análisis de varianza En este punto, se solucionó el problema 40 veces consecutivas (cada 10 soluciones se consideró un tratamiento) y en cada solución se tomó como referencia el mejor resultado. Los resultados obtenidos, en la variable tiempo total de proceso (Makespan), permiten construir el respectivo análisis de varianza. (Tabla 4).

Tabla 4. Análisis de varianza.

\begin{tabular}{|c|c|c|c|c|c|c|c|c|c|c|c|c|c|c|c|c|c|}
\hline $\begin{array}{l}\mathbf{T} \\
\text { Proceso }\end{array}$ & & & & & Rep & licas & & & & & Sum & Fuente Varia & G L & SC & CM & Fcal & \begin{tabular}{|l}
$\mathrm{F}$ \\
Tab \\
\end{tabular} \\
\hline $\begin{array}{l}\text { JSSP } \\
15 \times 20\end{array}$ & 1 & 2 & 3 & 4 & 5 & 6 & 7 & 8 & 9 & 10 & & Sum C Total & & 207,60 & & & \\
\hline Tratam 1 & 221 & 222 & 223 & 220 & 223 & 222 & 224 & 226 & 223 & 220 & 2224 & Tratamiento & 3,00 & 17,00 & 5,67 & 1,07 & 2,87 \\
\hline Tratam 2 & 220 & 223 & 221 & 222 & 226 & 227 & 221 & 224 & 226 & 227 & 2237 & Error Exper & 36,00 & 190,60 & 5,29 & & \\
\hline Tratam 3 & 224 & 226 & 227 & 224 & 222 & 221 & 223 & 220 & 224 & 227 & 2238 & Total & 39,00 & 207,60 & 10,96 & & \\
\hline Tratam 4 & 222 & 221 & 224 & 220 & 222 & 226 & 220 & 226 & 223 & 221 & 2225 & $\begin{array}{l}\text { EL MODELO } \\
\text { NO ES } \\
\text { SIGNIFICATIVO }\end{array}$ & & & & & \\
\hline
\end{tabular}

El análisis de varianza ilustrado en la Tabla 4, muestra que el valor de la distribución $F$, calculada en $(0,05 ; 3 ; 36)$, es menor que el valor de las mismas distribuciones $F$ reales, calculada en el mismo punto. En consecuencia se puede afirmar que los resultados obtenidos son estadísticamente iguales, con una confiabilidad de 99.5\%. Aspecto que establece la robustez del algoritmo.

Paso 6: Metodologías tradicionales. La tabla 5, muestra las soluciones del problema planteado por medio de las metodologías tradicionalmente empleadas: Operación más corta (OMC), Operación más larga (OML), Colas (FIFO), Pilas (LIFO) y Algoritmo de Campbell. 
Tabla 5. Aproximación de las metodologías tradicionales respecto al valor óptimo estimado.

\begin{tabular}{|l|l|l|l|}
\hline $\begin{array}{l}\text { Técnica } \\
\text { tradicional }\end{array}$ & $\begin{array}{l}\text { Tiempo de } \\
\text { proceso }\end{array}$ & $\begin{array}{l}\text { Tiempo } \\
\text { muerto }\end{array}$ & $\begin{array}{l}\text { Aproximación al } \\
\text { makespan óptimo } \\
\text { estimado. }\end{array}$ \\
\hline OMC & 297 & 2683 & $73.40 \%$ \\
\hline OML & 366 & 3718 & $59.56 \%$ \\
\hline FIFO & 309 & 2863 & $70.55 \%$ \\
\hline LIFO & 325 & 3103 & $67.07 \%$ \\
\hline Campbell & 337 & 3283 & $64.68 \%$ \\
\hline Promedio & $\mathbf{3 2 6 . 8}$ & $\mathbf{3 1 3 0}$ & $\mathbf{6 6 . 7 \%}$ \\
\hline
\end{tabular}

Un análisis de la Tabla 5, permite ver que en comparación con las técnicas tradicionales de programación de la producción y respecto a la variable tiempo total de proceso, la nueva metodología propuesta es superior en un $33 \%$.

\section{DISCUSIONES}

En las diversas revisiones literarias (Adil, et ál 2009; Nilgun, et ál. 2009; Louis, et ál 1996; Strusevich, 1997; Joachim 2001; Christian, 2005; Michel, et ál. 2008; Chianyao, et ál. 2009; López, et ál. 2009), se puede encontrar que el empleo de las técnicas de Inteligencia Artificial en la solución de problemas de secuenciación en ambientes Job Shop - Open Shop ha sido ampliamente difundido. Sin embargo, es importante resaltar que en este trabajo se muestra una metodología altamente efectiva (99.1\%), robusta (99.5\%) y con un tiempo de computación prácticamente despreciable (4 segundos). Aunque el tiempo de cómputo varía según la máquina de proceso, para esta metodología el mismo puede ser considerado despreciable, dado que el número máximo de iteraciones de la metodología propuesta es muy bajo (500).

Finalmente, en las revisiones literarias de este articulo, no se encontró un documento el cual condense los principales aspectos de las diferentes metodologías existentes (en la solución de problemas Job Shop - Open shop) en una nueva técnica y además contraste la efectividad de esta nueva técnica de inteligencia artificial, con las técnicas tradicionales de programación de la producción frente a un valor óptimo estimado.

\section{CONCLUSIONES}

Los resultados y discusiones, sobre la aplicación de un algoritmo evolutivo en la solución de un problema Job shop - Open shop, permiten obtener las siguientes conclusiones: 1) La nueva metodología propuesta logra obtener una solución que se aproxima a la solución óptima estimada en un porcentaje superior al 99\% con una robustez superior al $99.5 \%$ y un tiempo de computo prácticamente despreciable. 2) La metodología propuesta es fácil de replicar, pues para obtener los mismos resultados de este trabajo solo es necesario partir de una adecuada codificación inicial, como se describe en la figura 1, con las probabilidades de mutación y combinación genética descritas en este texto, bajo los operadores genéticos propuestos. 3) Los resultados obtenidos por la nueva metodología son altamente consistentes, tal como lo demuestra el análisis de varianza realizado. 4) En promedio, esta metodología mostro una superioridad del 33\% respecto a las técnicas tradicionales de programación de la producción, medida en la variables tiempo total de proceso 5) En futuras líneas de investigación, la metodología propuesta puede ser combinada con otras técnicas de inteligencia artificial como sistemas expertos, búsqueda tabú, minería de datos, etc. Las cuales permitirán mejorar los resultados obtenidos. 


\section{AGRADECIMIENTOS}

Este artículo es producto de la participación de sus autores en la investigación financiada por La Vicerrectoria de Investigaciones de la Universidad Nacional de Colombia y titulada "Secuenciación de la producción por medio de Algoritmos Evolutivos. Aplicación en ambientes Job Shop".

\section{REFERENCIAS}

Adil, B. y G. Mustafa, Gene Expression Programming based Due Date Assignment in a Simulated Job Shop, Expert Systems with Applications, 36 (10), 12143-12150 (2009).

Anaut, D,. et al. Optimización de redes eléctricas mediante la aplicación de algoritmos genéticos, Información Tecnológica, 20 (4), 137-148 (2009).

Castrillón, O., W. Sarache y J. Giraldo, Secuenciación en ambientes job shop por medio de agentes inteligentes y minería de datos, XII Congreso de Ingeniería de Organización, Burgos España. (2008).

Castrillón, O., W. Sarache y J. Giraldo, Solución de un problema Job shop por medio de un agente Inteligente, Ingeniería y Ciencia, 5 (10), 75-92 (2009).

Castrillón, O., W. Sarache y J. Giraldo, Técnicas inteligentes y estocásticas en scheduling, Un enfoque en la producción y las operaciones, Universidad Nacional de Colombia: Bogotá, Colombia (2010).

Christian, B. Beam-ACO-hybridizing ant colony optimization with beam search: an application to open shop scheduling, Computers \& Operations Research, 32 (6), 1565-1591 (2005).

Chao-Hsien, J. y H. Han-Chiang, A hybrid genetic algorithm for no-wait job shop scheduling problems, Expert Systems with Applications, 36 (3), 5800-5806 (2009).

Chinyao, L. y Yuling, Y. Genetic algorithm-based heuristics for an open shop scheduling problem with setup, processing, and removal times separated, Robotics and Computer-Integrated Manufacturing, 25 (2), 314-322 (2009).

De Giovanni, L. y F. Pezzella, An Improved Genetic Algorithm for the Distributed and Flexible Jobshop Scheduling problem, European Journal of Operational Research, 200 (2), 395-408 (2010).

Domínguez, M., S. Garcia., et ál, Dirección de Operaciones. Aspectos tácticos y operativos en la dirección de servicios. Mac Graw Hill: Madrid, España (1995).

Dy, S. y H. Cheng - Yu. Un enjambre de partículas nuevas de optimización para el problema de la programación de tienda abierta, Informática e investigación operativa, 35 (10), 3243-3261 (2008).

Koonce, D. A., Using data mining to find patterns in genetic algorithm solutions to a job Shob schedule, Computer \& Industrial Engineering, 38361 -374 (2000).

Latta, R. y G. Sarabia, Aplicación de la inteligencia artificial en sistemas automatizados de producción, Grupo de Ingeniería y Control TEISA, Universidad Cantabria, España (2000).

Leung, J., Handbook Of Scheduling, Boca Raton, Florida, USA (2004).

López, O., I. Ortega y V. Medina, A multi-agent system to construct production orders by employing an expert system and a neural network, Expert Systems with Applications, 36, 29372946 (2009).

Louis, S., Z. Xu. Genetic Algorithms for open shop scheduling and re-scheduling, Universidad de Nevada. (1996). 
Joachim, B., Günter, S., Vitaly A. Two-machine open shop scheduling with an availability constraint, Operations Research Letters, 29 (2), 65-77, 2001.

Michael, A., Heidemarie B., et ál. Simulated annealing and genetic algorithms for minimizing mean flow time in an open shop, 48 (7-8), 1279-1293 (2008).

Nebendahi. D., Sistemas Expertos: Introducción A La Técnica Y Aplicación, Marcombo S.A. Barcelona, España (2000).

Nilgun. F., O. Celal, et ál, Investigation of Ant System parameter interactions by using design of experiments for job-shop scheduling problems, Computers \& Industrial Engineering, 56, 538-559 (2009).

Pei-Chann, C., H. Jih-Chang, et ál, Artificial chromosomes embedded in genetic algorithm for a chip resistor scheduling problem in minimizing the makespan, Expert Systems with Applications, 36 7135-7141 (2009).

Pérez. S., Niño, Z. y Llobregat, M. Desarrollo de un programa de simulación de procesos para el tratamiento de emisiones atmosféricas, Información Tecnológica, 15 (2), 55-62 (2004).

Rancán, C., Arquitectura De Sistema Híbrido De Evaluación Del Alistamiento De Unidades Navales Auxiliares, Reportes Técnicos en Ingeniería de Software, 6 (1), 45-54 (2004).

Rohrer, M., Simulating of Manufacturing and Material Handling Systems, Procedente de Handbook of Simulation, Boca Raton, Florida, USA (2004).

Rosa, M., et al. Selección mediante simulación de una estructura de modelo cinético para la degradación aeróbica de efluentes de una industria láctea, Información tecnológica, 15 (2), 75-78 (2004).

Roshanaei, V., B. Naderi, et ál, A variable neighborhood search for job shop scheduling with setup times to minimize makespan, Future Generation Computer Systems, 25 (6), 654-661 (2009).

Savkin, A., J. Somlo, Optimal distributed real time scheduling of flexible manufacturing networks modeled as hybrid dynamical systems, Robotics and Computer-Integrated Manufacturing, 25, 597-609 (2009).

Strusevich, V., Hall., L. An open shop scheduling problem with a non-bottleneck machine, Operations Research Letters, 21 (1), 11-18 (1997).

Sierra, E., A. Hossian, et ál, Sistema experto para control inteligente de las variables ambientales de un edificio energéticamente eficiente, Universidad Nacional del Comahue, Buenos Aires, Argentina (2005).

Weiming, S., W. Lihui, H. Qi, Agent-Based Distributed Manufacturing Process Planning and Scheduling, A State-of-the-Art Survey And Reviews, 36 (4), (2006).

Zhang, Y., L. Xiaoping, Q. Wang, Hybrid genetic algorithm for permutation flowshop scheduling problems with total flowtime minimization, European Journal of Operational Research, 196, 869876 (2009). 